\title{
Mapping the Evolution of Grains in Strontium Titanate through Laboratory based 4D Diffraction Contrast Tomography
}

\author{
Amanda Krause ${ }^{1}$, Hrishikesh Bale ${ }^{2 *}$, Jun Sun ${ }^{3}$, William Harris ${ }^{2}$, Erik Lauridsen ${ }^{3}$, Christopher Marvel $^{5}$, \\ Carl Krill III ${ }^{4}$ and Martin Harmer ${ }^{5}$ \\ 1. University of Florida, Gainesville, FL, USA. \\ 2. Carl Zeiss X-ray Microscopy, Pleasanton, CA, USA. \\ 3. Xnovo Technology ApS, Køge, Denmark. \\ 4. Ulm University, Ulm, Germany. \\ ${ }^{5 .}$ Lehigh University, Bethlehem, PA, USA. \\ * Corresponding author: hrishikesh.bale@zeiss.com
}

Contrary to the commonly observed grain growth behavior in polycrystalline materials, some materials exhibit non-Arrhenius type growth behavior, where the grain growth rate decreases with increase in temperature [1]. This behavior, first referred to as 'anti-thermal behavior', was first observed in strontium titanate between $1350^{\circ} \mathrm{C}$ to $1425^{\circ} \mathrm{C}$ [2]. Such behavior is rather desirable in many high temperature applications and processing methods that strive to retain or achieve small grains. The underlying mechanisms that impart such unusual behavior are far more complex and remain elusive. Previously, the change in growth behavior has been correlated with changes in the grain boundary character distribution and grain boundary energy. However, the contribution of grain boundaries of different character, and their associated mobilities, to overall grain growth remains unknown.

The macroscopic grain boundary character can be identified with crystallographic imaging using electron back-scattered diffraction (EBSD), which is a well-established and routine tool for research and/or development related to metallurgy, functional ceramics, semi-conductors, geology etc. The ability to image the grain structure in materials is instrumental for understanding and optimization of material properties and processing. 3D-EBSD can provide sub-surface crystallographic information, but involves destructive sectioning of samples using focussed ion beams, which prevents any direct evaluation of microstructure evolution of the sample when subject to thermal, mechanical or other environmental conditions. This limits the ability to observe individual grain boundary motion, which is necessary to identify the critical grain boundaries for anti-thermal grain growth.

Non-destructive x-ray-based crystallographic imaging methods allow for extended '4D' time dependent studies but have to date been primarily the domain of a limited number of synchrotron facilities $[3,4]$. Recently laboratory-based diffraction contrast tomography (LabDCT) was introduced as an additional imaging modality on a commercially available X-ray microscope and thereby opened up a whole new range of possibilities for studies on the effect of 3D crystallography on materials performance [4-6]. LabDCT provides a full 3D crystallographic description of the grain boundary, allowing a comprehensive five parameter grain boundary characteristic analysis. The unique non-destructive 3D crystallographic imaging capabilities of LabDCT complement the structural data obtained by traditional absorption-based tomography and together they provide an unprecedented insight into the structure of materials linking information related to void/defect distribution and grain boundaries. In this study we report results obtained using LabDCT to track individual grains and grain boundaries of $\mathrm{SrTiO} 3$ at different timesteps during grain growth at two different temperatures. Here, we will discuss initial 
observations comparing growth at the two temperatures measured.

We will present reconstructed images from absorption and diffraction contrast tomography scans collected on a sintered, polycrystalline $\mathrm{SrTiO} 3$ sample. The absorption scans revealed the presence of significant porosity in the sample. LabDCT reconstructions reveal grains on that same sample. Spatially registering both these datasets enabled making investigation of the influence of voids on the grain boundaries. Results demonstrate that most voids lie in close proximity to grain boundaries. However a significant fraction of voids are also present within grains. In the 4D analysis we were able to track grains evolving over the two time steps. Furthermore, the technique's opportunities and challenges for studying anti-thermal grain growth will be discussed.

References:

[1] PR Cantwell et al., Scripta Materialia 103 (2015), p. 1.

[2] M Bäurer et al., Scripta Materialia 61 (2009), p. 584.

[3] HF Poulsen et al., MRS Bull. 29(3) (2004), p. 166.

[4] W Ludwig et al., J. Appl. Crystallogr. 41(2) (2013), p. 310.

[5] SA McDonald et al., Sci Reports 5 (2015), p. 14665.

[6] F Bachmann et al., "3D grain reconstruction from LabDCT”, submitted.
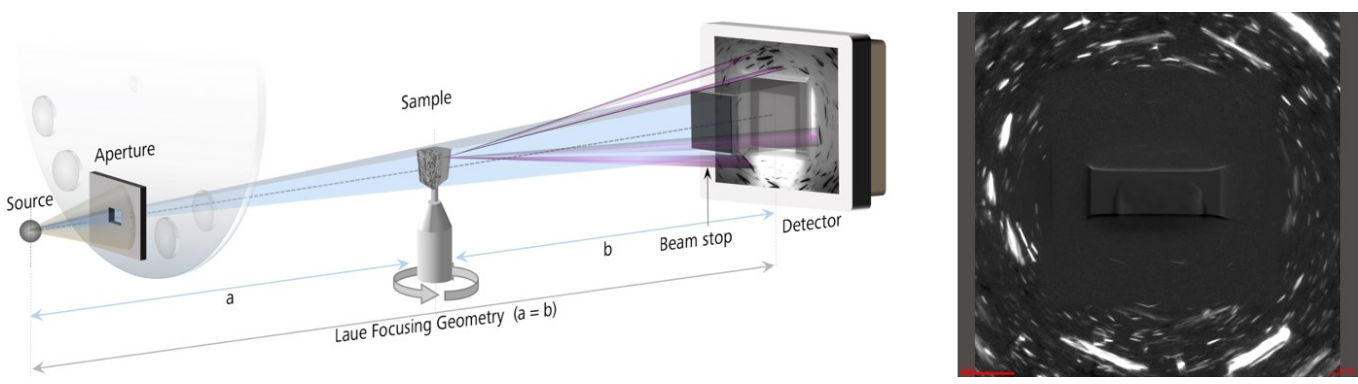

Figure 1. (a) Schematic showing the experimental setup of LabDCT in the laboratory X-ray microscope. (b) Raw diffraction pattern from $\mathrm{SrTiO3}$ sample; large reflections correspond to the abnormal grain at the top.

(a)

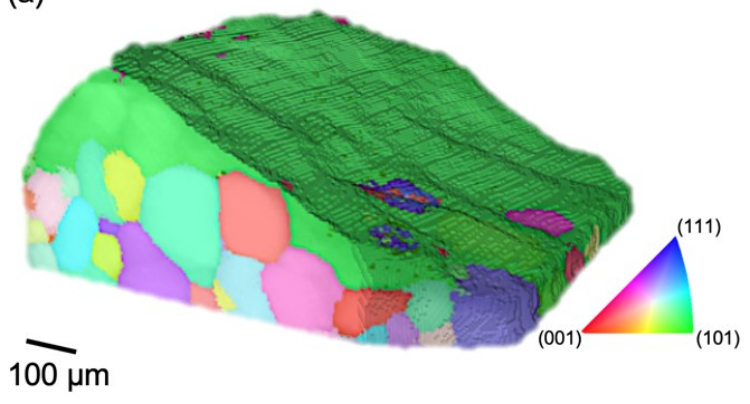

(b)

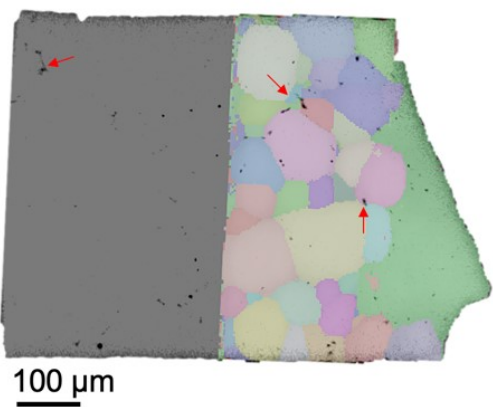

Figure 2. (a) 3D grain reconstruction from LabDCT indicated in the IPF color scheme for the 100 direction. (b) overlaid LabDCT and absorption tomography results. Voids are clearly seen dispersed along grain boundaries. 